\title{
Impact du tritium autour des centrales nucléaires EDF
}

\author{
B. LE GUEN ${ }^{1}$
}

Une faute éditoriale s'est insérée dans le texte publié dans :

Radioprotection 2008, Vol. 43, $n^{\circ}$ 2, pages 177 à 191

l'unité microsievert a malencontreusement été remplacée par mSv en particulier:

\section{Page 189, il faut lire :}

La dose totale calculée à partir des rejets liquides de l'année 2004 (ensemble des radionucléides) varie entre 0,2 microsievert par an à Flamanville et 1 microsievert par an à Gravelines. Le tritium sous forme OBT intervient à hauteur de $15 \%$ dans ce calcul de dose.

Pour un site marin, les quantités de produits de la mer ingérées peuvent néanmoins être plus élevées pour certains groupes socioprofessionnels comme les pêcheurs. C'est pourquoi, des calculs ont également été effectués à la fois pour le groupe de référence et pour ce groupe nommé « groupe pêcheur » différent du groupe de référence.

Pour exemple sur le site de Flamanville, l'étude d'impact réalisée pour les demandes d'autorisations de rejets pour le site EPR estime que les doses dues au tritium liquide, en tenant compte des «rejets maximaux», est évaluée à environ 0,014 microsievert/an pour le « groupe de référence » $(0,008$ microsievert (tranches $1-2)+$ 0,006 microsievert (EPR)) et 0,037 microsievert par an pour le «groupe pêcheur », $(0,022$ microsievert (tranches 1-2) + 0,015 microsievert (EPR)).

\section{Page 190, il faut lire :}

Par calcul empirique, on estime que, sur une année, une personne mangeant du poisson péché à proximité d'une centrale comme celle de Flamanville, qui se baignerait régulièrement avec passage cutanée du tritium, et qui boirait jusqu'à 20 litres d'eau de mer tritiée lors de ses baignades (en évitant la noyade !), pourrait intégrer une dose équivalente à 4 millionième de la limite annuelle pour le public $(=0,004 \underline{\text { microsievert }})$.

\footnotetext{
1 Conseil de radioprotection EDF (CRP) et EDF, DIN, CEIDRE, 2 rue Ampère, 93206 Saint-Denis Cedex 1, France.
} 